\title{
Collision-Induced Absorption of Hydrogen Deuteride Dissolved in Liquid Neon
}

\author{
W. Herrebout, ${ }^{1}$ B. Van der Veken, ${ }^{1}$ A. P. Kouzov, ${ }^{2, *}$ and M. O. Bulanin ${ }^{2}$ \\ ${ }^{1}$ University of Antwerpen, RUCA, Antwerpen, Belgium \\ ${ }^{2}$ Institute of Physics, Saint Petersburg State University, Peterhof, Saint Petersburg 198504, Russia
}

(Received 22 August 2003; published 15 January 2004)

\begin{abstract}
First observation of the collision-induced IR-fundamental band of hydrogen deuteride (HD) in liquid neon is described. A developed intracell rattling model yields highly accurate fits to the measured diffuse $Q_{1}$-branch profiles enabling a detailed shape analysis of the adjacent spectral lines. Strong intracollisional anticorrelation found at the location of the permanent dipole-allowed $R_{1}(0)$ transition is interpreted in the frame of the Fano-Mori theory. A new striking narrowing effect (by $30 \%$ ) is observed on the sharp $Q_{1}^{q}(0)$ vibrational line with increasing HD concentration in solutions. The $T_{1}(0), U_{1}(0)$ and a few pair transitions are identified in the spectrum.
\end{abstract}

PACS numbers: 33.20.Ea, 34.90.+q, 61.25.Bi

Interaction-induced vibration-rotation-translation spectra of molecular hydrogen furnish a unique opportunity to study translational motion and its coupling to the internal degrees of freedom of molecules in high-density phases [1,2]. Further unusual effects appear in the spectra of hydrogen deuteride (HD) because the center of mass in this molecule is shifted from the bond midpoint. This results in an extra torque exerted on the HD molecule from neighboring particles that makes its rotation stronger perturbed compared to symmetric $\mathrm{H}_{2}$ and $\mathrm{D}_{2}$ and reveals more detail on the molecular motion dynamics in condensed media. The lack of the center-of-mass inversion symmetry for HD renders active the rotational transitions with $\Delta J=$ odd [3]. Because of the intramolecular electronic-rotovibrational coupling, HD acquires a small permanent dipole moment $M_{a}$, comparable in magnitude with the typical values of the collisioninduced dipole moments $\mu_{a b}$. The intracollisional correlation (IC) between these dipoles distorts the allowed $P_{1}$ and $R_{1}$ spectral line profiles [4] mostly studied so far in the gas-phase spectra (see, e.g., [5,6], and references therein). Similar effects were observed in the liquid state (neat HD [7] and its solution in Ar [3]), but no theoretical interpretation was advanced.

We report here new data on the structure of the previously unexplored fundamental absorption band of HD dissolved in liquid neon at different temperatures and concentrations. By varying the HD concentration $x_{\mathrm{HD}}$ in solution one can identify spectral features induced by the guest-host and guest-guest interactions, the latter being responsible for the appearance of the so-called simultaneous transitions (ST) resulting in excitation of a pair of interacting HD molecules by a single photon. The excited rotational states become depopulated at low temperatures of our experiments, which simplifies the spectral band structure and favors resolution of finer details. On the other hand, the Ne solvent density changes in the temperature range covered are large enough [8] to significantly modify both the frequency distribution and the integrated band intensity.
A remarkable narrowing of the $Q_{1}^{q}(0), \Delta J=0$ vibrational spectral line was found upon increase of the solute concentration in solution; to the best of our knowledge, no such self-induced line narrowing effect was observed before. To separate this and the permanent dipole-allowed $R_{1}(0)$ spectral features from the diffuse collision-induced $Q_{1}$-branch background, the background shape is fitted using a simple cage model of liquid. A general FanoMori approach to the IC shape theory is outlined.

The spectra were recorded at $0.5 \mathrm{~cm}^{-1}$ resolution with a Bruker IFS 66v FTIR interferometer in combination with a $\mathrm{Ge} / \mathrm{KBr}$ beam splitter, a Globar source, and a broadband MCT detector, by averaging 1000 scans and applying the Blackman-Harris apodization. Gas samples of HD (Isotec, stated purity 98\%) and $\mathrm{Ne}$ (Air Liquide, stated purity $99.995 \%$ ) were used without further purification. Premixed gases were condensed in a sample cell attached to the cold head of a Leybold ROK 10-300 double stage cryostat. The cell of $4 \mathrm{~cm}$ pathlength, made of brass and designed to withstand internal pressure of at least $30 \mathrm{bar}$, was equipped with a pair of $1^{\circ}$-wedged silicon windows sealed by indium gaskets and with two $\mathrm{Si}$ diodes located at the top and the bottom of the cell body for temperature control in the range of $24-43 \mathrm{~K}$. The setup did not allow us to control whether all HD from the gas mixtures was actually dissolved in liquid neon, so that no measurements of the absolute spectral intensities were attempted. The nominal $x_{\mathrm{HD}}$ values were in the 0.01-0.04 mole fraction (m.f.) range.

Spectral assignment.-Some of the recorded spectra are shown in Figs. 1 and 2. A surprisingly sharp $Q_{1}^{q}(0)$ feature appears at the pure vibration frequency $\nu_{0}=$ $\omega_{0} / 2 \pi c=3632.1 \mathrm{~cm}^{-1}$ (all quoted free-molecule frequencies are taken from Ref. [9]). A prominent asymmetric dip is located at the position of the allowed $R_{1}(0)$ line at $\nu=3717.5 \mathrm{~cm}^{-1}$. At higher temperatures, a similar dip develops near the $P_{1}(1)$ line, at $\nu=3542.9 \mathrm{~cm}^{-1}$, and a weak absorption peak due to the $R_{1}(1)$ transition, $\nu=3798.4 \mathrm{~cm}^{-1}$, becomes visible on the slope of the broad $Q_{1}^{r}$ band. The unresolved doublet band near 


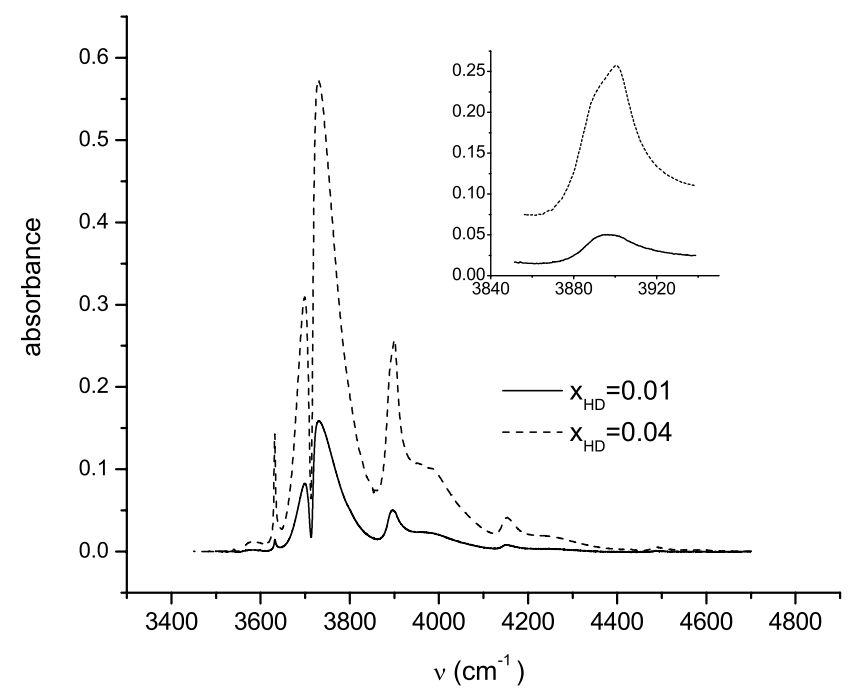

FIG. 1. HD fundamental absorption band at $T=25.7 \mathrm{~K}$ at two different HD concentrations. The inset shows the enlarged part of the spectrum near the $S_{1}(0)$ transition.

$3900 \mathrm{~cm}^{-1}$ observed at $x_{\mathrm{HD}}=0.04$ m.f. is formed by the induced $S_{1}(0)$ single transition at $3887.6 \mathrm{~cm}^{-1}$ and overlapping ST $Q_{1}(0)+S_{0}(0), \nu=3899.2 \mathrm{~cm}^{-1}$, that becomes undetectable in the spectrum of a more dilute solution. A broad shoulder near $4000 \mathrm{~cm}^{-1}$ is most likely the $S_{1}^{r}$ intracell rattling component. Next are the $\Delta J=3$ $\left[T_{1}(0)\right.$ line at $\left.4141.3 \mathrm{~cm}^{-1}\right]$ and $\Delta J=4\left[U_{1}(0)\right.$ line at $4476.7 \mathrm{~cm}^{-1}$ ] transitions. Their blue wings overlap with the positions of the $S_{1}(0)+S_{0}(0) \quad\left(\nu=4154.3 \mathrm{~cm}^{-1}\right)$, $Q_{1}(0)+T_{0}(0) \quad\left(\nu=4164.5 \mathrm{~cm}^{-1}\right)$ and $Q_{1}(0)+U_{0}(0)$ $\left(\nu=4515.4 \mathrm{~cm}^{-1}\right)$ lines. However, no concentration effects on the blue wing distributions were detected and, hence, these STs have negligible intensities. Except $Q_{1}(0)+S_{0}(0)$, the only ST observable feature is $S_{1}(0)+$ $T_{0}(0), \nu=4419.6 \mathrm{~cm}^{-1}$ which becomes visible in the $x_{\mathrm{HD}}=0.04$ m.f. solution. It rapidly fades away with increasing temperature (see inset to Fig. 2). To make the absorbances on the inset approximately equal, they were rescaled by normalizing the spectra by the unity area.

Separation of components near the band origin.The spectral density function $W(\omega)$ is obtained from the measured absorbance $A(\omega)$ by $W(\omega)=$ $\left(\omega_{0} / \omega\right)[1-\exp (-\hbar \omega / k T)] A(\omega)$. Within the experimental uncertainty, the Boltzmann relation $W(-\Delta \omega)=$ $W(\Delta \omega) \exp (-\hbar \Delta \omega / k T)$ holds relative to the band origin, i.e., by taking $\Delta \omega=\omega-\omega_{0}$. This implies that the translational motion of solutes remains decoupled from molecular vibrations and, possibly, from rotations as well, as suggested by the quantum asymmetry apparent on the partially resolved $T_{1}(0)$ and $U_{1}(0)$ spectral profiles (see Figs. 1 and 2,).

We assume HD solutes to make one-dimensional oscillations with the frequency $\omega_{r}$ between the cage walls formed by the Ne atoms. Since the intensity of the diffuse part of the $Q$ branch originates from the short-range

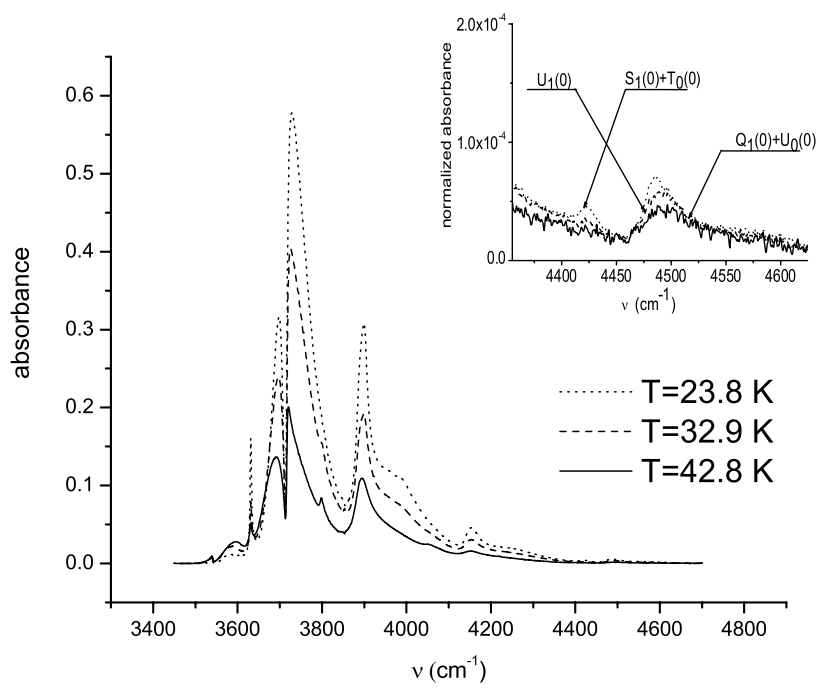

FIG. 2. Fundamental band of $\mathrm{HD}$ in liquid neon at $x_{\mathrm{HD}}=$ 0.04 m.f. Inset: $U_{1}(0)$ spectral feature; arrows indicate the transition frequencies.

terms of the induced dipole moment function, one may expect the HD molecule to effectively interact with a single $\mathrm{Ne}$ atom during impact with the wall. In that case, the impact time correlation function $C_{0}(t)$ will have the same form as for the isolated gas-phase binary collisions with the mean duration $t_{c}=\omega_{c}^{-1}$. Finally, intracell rattling and wall collisions are considered as statistically independent events, which yields the shape function $W_{s}(\Delta \omega)=\left[W_{0}\left(\Delta \omega+\omega_{r}\right)+W_{0}\left(\Delta \omega-\omega_{r}\right)\right] / 2$, where $W_{0}(x)$ is the Fourier transform of $C_{0}(t)$. The model function we are using is $W_{0}(x)=A \exp \left(\lambda-\left[\lambda^{2}+\right.\right.$ $\left.\left.\left(x / \omega_{c}\right)^{2}\right]^{1 / 2}\right)$, previously shown [10] to accurately fit the shapes of the translational absorption in rare-gas mixtures, also induced by the short-range interactions [11]. The $\lambda$ parameter is temperature insensitive, so a fixed value of $\lambda=1.5$ was taken [10]. Next, a correcting factor $D(\Delta \omega)=1-d \exp \left[-\left(\Delta \omega / \omega_{r}\right)^{2} / 2\right]$ was introduced to improve the quality of fits near $\omega_{0}$. The symmetrized four-parametric $\left(A, \omega_{c}, \omega_{r}, d\right)$ model shape function $W(\Delta \omega)$ then becomes

$$
\begin{aligned}
\tilde{W}(\Delta \omega) & \equiv[W(\Delta \omega)+W(-\Delta \omega)] / 2 \\
& =W_{s}(\Delta \omega) D(\Delta \omega) E(\Delta \omega),
\end{aligned}
$$

where $E(\Delta \omega)=\cosh (\hbar \Delta \omega / 2 k T)$ corrects for the quantum effects.

The fittings were performed in the frequency ranges where contributions due to the $R_{1}(0)$ and $Q_{1}^{q}(0)$ features are negligible and produced profiles indiscernible from the experimental data. Since the shapes of the diffuse collision-induced $Q$ branches are smooth, one may interpolate $W(\Delta \omega)$ to obtain the $R_{1}(0)$ and $Q_{1}^{q}(0)$ line profiles as differences between the measured and simulated distributions. The inverse collision time $\omega_{c}$ should increase 
with $T$ (classically as $\sqrt{T}$ ); indeed, the corresponding frequency factors $\left(\omega_{c} / 2 \pi c\right)$ were found to increase from $21 \mathrm{~cm}^{-1}$ at $T=23.8 \mathrm{~K}$ up to $29 \mathrm{~cm}^{-1}$ at $T=42.8 \mathrm{~K}$. From the latter value one can estimate [10] the induceddipole range parameter, $R_{0}=0.72$ a.u., a value consistent with the short-range nature of the dipole moment responsible for the appearance of the diffuse $Q_{1}$-branch component. The rattling frequency $\omega_{r}$ depends on the cage radius $a\left(\omega_{r} \sim a^{-2}\right)$. In turn, $a$ depends on the solvent density $\rho_{b}$, which is strongly subjected to temperature [8]. For instance, at outer pressure of $20 \mathrm{~atm}$, one gets from Ref. [8]: $\rho_{b}=1416$ Amagat $(T=23.8 \mathrm{~K})$ and $\rho_{b}=$ 902 Amagat $(T=42.8 \mathrm{~K})$. The rattling slows down as more space for HD motion becomes available, indeed $\omega_{r} / 2 \pi c$ was found to decrease from $73 \mathrm{~cm}^{-1}$ at $T=$ $23.8 \mathrm{~K}$ to $40 \mathrm{~cm}^{-1}$ at $T=42.8 \mathrm{~K}$.

Intracollisional shape.-An unusual feature observed in the spectrum of the HD-Ne system is that the $R_{1}(0)$ and $P_{1}(1)$ transitions appear not as ordinary absorption peaks, but as asymmetric dips on the $Q_{1}$-branch background. The dip asymmetry is enhanced with increasing temperature and a positive bump appears at the blue side of the $R_{1}(0)$ feature (see Fig. 3). A similar but far less developed pattern was previously observed in pure gaseous HD and its mixtures with rare gases in both pure rotational [12] and rotovibrational spectra [13].

We treat cross correlation between the permanent dipole $M_{a}$ of a particular HD molecule and the net induced dipole moment $\mu^{\Sigma}=\sum_{b} \mu_{a b}$ by applying the Fano-Mori technique [14]. The molecule is characterized by a set of the allowed rotovibrational transitions $I \rightarrow F$ associated with the line-space vector $\left.|K\rangle\rangle=\rho_{a I}^{-1 / 2}\left|I F^{\times}\right\rangle\right\rangle$and the matrix elements $M_{K}=\sqrt{\rho_{a I}}\left\langle I\left|M_{a}\right| F\right\rangle$, where $\rho_{a}$ is the molecular density matrix. The collision-induced dipole $\left.\mu^{\Sigma}=\left|\mu^{\Sigma}(q)\right\rangle\right\rangle$ depends also on a set of the translational coordinates $q$. With the help of two projectors $\hat{P}$ and $\hat{Q}=$

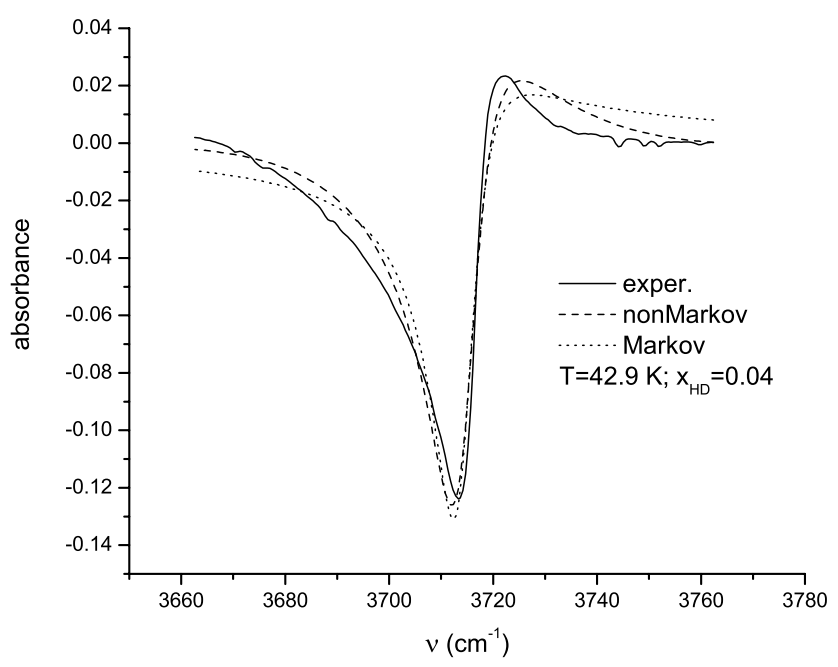

FIG. 3. Intracollisional $R_{1}(0)$ shape.
$1-\hat{P}$, it can be decomposed into two orthogonal (in the sense of the line-space metric [14]) components, one $\left.\left(\left|\mu_{P}\right\rangle\right\rangle\right)$ varying in-phase with $M_{a}$, and the other $\left.\left(\left|\mu_{Q}\right\rangle\right\rangle\right)$ corresponding to the rapidly fluctuating part of the collision-induced dipole. The in-phase component when added to $M_{a}$, simply rescales line intensities in the allowed spectrum as $M_{K}^{2}\left(1+f_{K}\right)^{2}$ via the real, densitydependent coefficients $f_{K}=M_{K}^{-1}\left\langle\left\langle K \mid \mu_{I F}^{\Sigma}\right\rangle\right\rangle$.

In our case the allowed lines are well apart from each other, so the self-part of the complex spectral function near $\omega_{K}=\omega_{F I}$ is given by

$$
S_{\text {self }}(\omega)=\frac{i}{\pi} \frac{M_{K}^{2}\left(1+f_{K}\right)^{2}}{\omega-\Omega_{K}+i \Gamma_{K K}}+S_{Q}(\omega),
$$

where $S_{Q}(\omega)$ is the background spectrum associated with $\mu_{Q}$. The "dressed" frequency $\Omega_{K}$ may differ from $\omega_{K}$ because of the bath-molecule coupling Liouvillian $\hat{L}_{1}$. The complex damping parameter $\Gamma_{K K}=\Gamma_{K K}^{\prime}+i \Gamma_{K K}^{\prime \prime}$ is determined by the self-spectrum of $\left.\left|L_{1} K\right\rangle\right\rangle$ [14]. The derived cross term

$$
S_{\text {cross }}(\omega)=\frac{1}{\pi} \frac{M_{K}^{2}\left(1+f_{K}\right) \Phi_{K}}{\omega-\Omega_{K}+i \Gamma_{K K}} .
$$

contains the factor $\Phi_{K}$ defined by the cross-correlation spectrum of rapidly decaying fluctuations $\left.\left|L_{1} K\right\rangle\right\rangle$ and $\left|\mu_{Q}\right\rangle$.

$$
\begin{aligned}
\Phi_{K}= & \left\langle\left\langle K\left|L_{1}^{\dagger} Q \frac{i}{\omega-L Q}\right| \mu_{Q}\right\rangle\right\rangle \\
& +\left\langle\left\langle\mu_{Q}\left|Q \frac{i}{\omega-L Q} L_{1}\right| K\right\rangle\right\rangle,
\end{aligned}
$$

where $\hat{L}$ is the total Liouvillian. For detunings from $\omega_{K}$ much smaller than the inverse time $t_{c}^{-1}$ of the fluctuation decay, $\Phi_{K}$ and $\Gamma_{K}$ do not depend on $\omega$. Such detunings correspond to the Markovian collisions in the gas phase.

The total absorption profile is obtained as the real part of $S_{\text {self }}(\omega)+S_{\text {cross }}(\omega)$. In the Markovian limit, it reduces to the Fano profile with an asymmetric dispersion term determined by $\Phi_{K}^{\prime}=\operatorname{Re} \Phi_{K}$. The imaginary part $\Phi_{K}^{\prime \prime}=$ $\operatorname{Im} \Phi_{K}$ contributes to the integrated intensity $I_{K}=$ $M_{K}^{2}\left(1+f_{K}\right)\left(1+f_{K}+\Phi_{K}^{\prime \prime}\right)$, which-dependent on the values of $f_{K}$ and $\Phi_{K}^{\prime \prime}$ - can be either positive or negative. The signs of $\Gamma_{K K}^{\prime}$ and $\Phi_{K}^{\prime}$ depend on the relevant initialtime correlations: obviously, $\Gamma_{K K}^{\prime}(\omega)>0$ and the sign of $\Phi_{K}^{\prime}(\omega)$ coincides with that of $M_{K}\left\langle\left\langle\mu_{Q}\left|L_{1}\right| K\right\rangle\right\rangle$ irrespective of the frequency. The signs of $\Gamma_{K}^{\prime \prime}$ and $\Phi_{K}^{\prime \prime}$ cannot be easily predicted. Since $\mu_{I F}^{\Sigma}$ is given by the sum of binary contributions, $f_{K}$ is proportional to the solvent density $\rho_{b}$ and only small deviations from the linear dependence are possible. In the case of $\Phi_{K}$ and $\Gamma_{K K}$, the linear law holds only under the binary-collision regime.

Except the neglect of line mixing, the obtained results are rigorous and five independent real constants $\left(f_{K}, \Gamma_{K K}^{\prime}, \Omega_{K}+\Gamma_{K K}^{\prime \prime}, \Phi^{\prime}{ }_{K}, \Phi_{K}^{\prime \prime}\right)$ specify the spectral profile in the Markovian limit. However, only four 
combinations $p_{k}(k=1,2,3,4)$ of these constants can be extracted from the measurement:

$$
\begin{aligned}
& p_{1}=\frac{M_{K}^{2}\left(1+f_{K}\right)\left(1+f_{K}+\Phi_{K}^{\prime \prime}\right)}{\pi \Gamma_{K K}^{\prime}}, \quad p_{2}=\Gamma_{K K}^{\prime}, \\
& p_{3}=\Omega_{K}-\Gamma_{K K}^{\prime \prime}-\omega_{f i}, \quad p_{4}=\frac{\Phi_{K}^{\prime}}{\left(1+f_{K}+\Phi_{K}^{\prime \prime}\right)} .
\end{aligned}
$$

Furthermore, only the sign of $p_{1}$ is physically meaningful because the absolute intensities have not been measured in the present study. The gas-phase data for HD-Ne at $T=$ $77 \mathrm{~K}$ [13] suggest negative scaling: $1+f_{K}<0$. Since $p_{1}$ and $p_{4}$ remain negative at any temperature, we conclude that $\Phi_{K}^{\prime \prime}+1+f_{K}>0, \Phi_{K}^{\prime}<0$; the latter implies that $M_{K}\left\langle\left\langle\left\langle\mu_{Q}\left|L_{1}\right| K\right\rangle\right\rangle<0\right.$.

As Fig. 3 shows, the Markovian fit systematically overestimates the intensity in the $R_{1}(0)$ dip wings. This is caused by a too slow decay (as $|\Delta \omega|^{-1}$ ) of the dispersion component at large detunings. Therefore, some nonMarkovian correction should be introduced first for $\Phi^{\prime}(\Delta \omega)$. For instance, the use of a Gaussian function for $\Phi^{\prime}(\Delta \omega)$ indeed improves the quality of the fit to the experimental profile.

The $J=1$ solute state begins to be noticeably populated at $T=42.8 \mathrm{~K}$ and the $P_{1}(1)$ and $R_{1}(1)$ transitions become observable. Although their recorded intensities were too small for a quantitative treatment, two features can be noted. First, the $P_{1}(1)$ dip profile is qualitatively a reflection at the band origin of the $R_{1}(0)$ profile, indicating that $\Phi_{P_{1}(1)}^{\prime}>0$. This result suggests that the rotational contributions to $\Phi_{P_{1}(1)}^{\prime}$ and $\Phi_{R_{1}(0)}^{\prime}$ are dominating. These two transitions have inverted initial and final rotational quantum numbers and such inversion changes the sign of $\Phi_{K}^{\prime}$, in agreement with observed behavior. Second, the IC effects on the $R_{1}(1)$ shape are constructive and less pronounced compared to the $R_{1}(0)$ case, as was also observed in the spectrum of a compressed HD-Ne gas mixture at $T=77 \mathrm{~K}$.

Vibrational $Q_{1}^{q}(0)$ line.-This sharp line must be induced by the "isotropic" part of $\mu_{a b}$ insensitive to molecular rotation. The dispersion contribution, $\mu_{a b} \sim$ $D_{7} R^{-7}$, seems to be the most likely candidate. After separation from the diffuse $Q_{1}$-branch background, its shape appears as a Lorentzian shifted by $0.7 \mathrm{~cm}^{-1}$ to lower frequencies compared to the gas phase [9]. Since the solute rotation is not involved, the $Q_{1}^{q}(0)$ line halfwidth $\gamma$ reflects the combined effect of translational and vibrational degrees of freedom. Temperature variation of $\gamma(\mathrm{HWHH})$ is linear, increasing from $2.0(1) \mathrm{cm}^{-1}$ at $23.8 \mathrm{~K}$ to $4.4(3) \mathrm{cm}^{-1}$ at $42.8 \mathrm{~K}$ in the $x_{\mathrm{HD}}=0.04$ m.f. solution. These values are about 3 times smaller than the $R_{1}(0)$ half-width, which is dependent also on the rotational relaxation. The area under the $Q_{1}^{q}(0)$ line $A(T=$ $25.7 \mathrm{~K}$ ) increased by more than sevenfold in the spectrum of the solution with $x_{\mathrm{HD}}=0.04$ m.f. compared to that observed in the $x_{\mathrm{HD}}=0.01$ m.f. solution. The measured values fit the equation $A\left(x_{\mathrm{HD}}\right)=A_{1} x_{\mathrm{HD}}+A_{2} x_{\mathrm{HD}}^{2}$ with $A_{1}=7.5 \mathrm{~cm}^{-1}$ and $A_{2}=320 \mathrm{~cm}^{-1}$ showing that both HD monomers and dimers contribute in the induction of this spectral line.

The most striking observation is a strong concentration narrowing of the $Q_{1}^{q}(0)$ line. Its HWHH $(T=25.7 \mathrm{~K})$ decreased from $3.2(1) \mathrm{cm}^{-1}$ at $x_{\mathrm{HD}}=0.01 \mathrm{~m}$.f. to $2.1(1) \mathrm{cm}^{-1}$ at $x_{\mathrm{HD}}=0.04$ m.f. Pure translational narrowing mechanism can hardly apply because HD concentrations were too small to perturb so strongly the solvent motion. One may consider the vibrational perturbations as the most probable cause, however, to explain how the combined action of dephasing and vibrational energy transfer could produce a net line narrowing remains unclear and requires further study.

Financial support from the Flemish Community (Special Research Fund-BOF), the FWO-Vlaanderen, and the Russian Foundation for Basic Research is gratefully acknowledged.

*Corresponding author.

Email address: alex@apk.usr.pu.ru

[1] J. van Kranendonk, Solid Hydrogen (Plenum Press, New York, 1983).

[2] Molecular Cryospectroscopy, edited by M. O. Bulanin, Advances in Spectroscopy Vol. 23, edited by R. J.H. Clark and R.E. Hester (Wiley \& Sons, Chichester, 1995), Ch. 6.

[3] G.W. Holleman and G. E. Ewing, J. Chem. Phys. 44, 3121 (1966); 47, 571 (1967).

[4] R. M. Herman, R. H. Tipping, and J. D. Poll, Phys. Rev. A 20, 2006 (1979).

[5] M. Gustafsson and L. Frommhold, Phys. Rev. A 63, 052514 (2001).

[6] A. R.W. McKellar, Can. J. Phys. 64, 227 (1986).

[7] M. J. Clouter and A. R.W. McKellar, Can. J. Phys. 65, 1 (1987).

[8] Handbook on Thermophysical Properties of Gases and Liquids, edited by N.B. Vargaftik (Nauka, Moscow, 1979).

[9] B. P. Stoicheff, Can. J. Phys. 35, 730 (1957).

[10] A. P. Kouzov, Opt. Spektrosk. 30, 841 (1971).

[11] U. Buontempo, S. Cunsolo, and G. Jacucci, Can. J. Phys. 49, 2870 (1971).

[12] A. R.W. McKellar, J.W. C. Johns, W. Majevski, and N. H. Rich, Can. J. Phys. 62, 1673 (1984).

[13] A. R.W. McKellar and N. H. Rich, Can. J. Phys. 62, 1665 (1984).

[14] D. Forster, Hydrodynamic Fluctuations, Broken Symmetry and Correlation Functions (Benjamin, Massachusetts, 1975). 